\title{
Influencing Social Networks for P2P Substance-based File Distribution in Disjointed MANETs
}

\author{
S.Palani \\ MCA.,M.E Assistant Professor, \\ Department of MCA, \\ Sri Venkateswara College of \\ Engineering \& Technology, \\ Chittoor
}

\author{
Sendhil Kumar, \\ M.E \\ Associate Professor, \\ Department of MCA, \\ Sri Venkateswara College of \\ Engineering \& Technology, \\ Chittoor
}

\author{
P.Madhuri \\ PG Scholar, \\ Department of MCA, \\ Sri Venkateswara College of \\ Engineering \& Technology, \\ Chittoor
}

\begin{abstract}
Conceptual Current shared (P2P) document imparting routines in versatile specially appointed systems (MANETs) can be ordered into three gathering flooding-based, notice based, and social contact-based. The initial two gatherings of strategies can without much of a stretch have high overhead and low adaptability. They are primarily created for joined MANETs, in which end-to-end integration among hubs is guaranteed. The third gathering of routines adjusts to the entrepreneurial way of detached MANETs however neglects to consider the social hobbies (i.e., substance) of portable hubs, which can be abused to enhance the record seeking effectiveness. we propose a $\mathrm{P} 2 \mathrm{P}$ content based document imparting framework, to be specific SPOON, for detached MANETs. The framework utilizes an interest extraction calculation to infer a hub's hobbies from its records for substance based document looking. For productive document looking, SPOON bunches regular interest hubs that often times meet with one another as groups. It exploits hub versatility by assigning stable hubs, which have the most incessant contact with group individuals, as group facilitators for intra community seeking, and exceedingly portable hubs that visit different groups regularly as group envoys for intercommunity seeking. An interestarranged document seeking plan is proposed for high record seeking productivity. Extra methods for document prefetching, questioning finishing, and loop-prevention, also, hub agitate thought are examined to further upgrade the record seeking productivity. We initially tried our framework on the GENI Orbit testbed with a genuine follow and afterward directed occasion driven examination with two genuine follows and NS2 reenactment with mimicked disengaged and joined MANET situations. The test outcomes demonstrate that our framework fundamentally brings down transmission cost.
\end{abstract}

\section{General Terms}

SPOON, GENI, MANET

\section{Keywords}

MANETs, Social Networks, Substance-based file distribution.

\section{INTRODUCTION}

The previous couple of years, individual cell phones, for example, tablets, PDAs, and cell phones have been more and more mainstream. Without a doubt, the quantity of cell phone clients expanded by 118 million over the world in 2007, and is anticipated that would stretch around 300 million by 2013 . The fantastically quick development of portable clients is prompting a promising future, in which they can uninhibitedly impart documents between one another at whatever point and wherever. The quantity of versatile seeking clients (through cell phones, highlight telephones, tablets, and so forth.) is evaluated to achieve 901.1 million in 2013. Right now, portable clients connect with one another furthermore; impart documents through a foundation shaped by geologically circulated base stations. Notwithstanding, clients may discover themselves in a region without remote administration (e.g., mountain regions and provincial territories). Besides, clients might want to lessen the expense on the extravagant base system information. The P2P document offering model makes huge scale organizes a gift rather than a condemnation, in which hubs offer documents specifically with one another without a unified server also, enhances record looking achievement rate contrasted with current systems. Wired P2P record offering frameworks have as of now turned into a prevalent and fruitful ideal model for record offering among a large number of clients. The effective organization of P2P document imparting frameworks and the previously stated hindrances to document partaking in MANETs make the P2P document offering over MANETs (P2P MANETs in short) a promising supplement to current framework model to acknowledge pervasive document offering for versatile clients. As the portable advanced gadgets are conveyed by individuals that generally fit in with certain social connections, in this paper, we center on the P2P document partaking in a detached MANET group comprising of portable clients with informal organization properties. In such a document offering framework, hubs meet and trade demands and documents in the arrangement of content, short features, and voice cuts in distinctive interest classifications. A common situation is a course material (e.g., course slides, survey sheets, assignments) imparting framework in a school grounds. Such a situation guarantees for the most that hubs having the same hobbies (i.e., math), convey comparing documents (i.e., math records), and meet routinely (i.e., going to math classes).

In MANETs comprising of computerized gadgets, hubs are always moving, shaping disengaged MANETs with deft hub experiencing. Such transient system associations have represented a test for the improvement of P2P MANETs. Customary systems supporting P2P MANETs are either flooding-based or commercial based. 


\section{SYSTEM ARCHITECTURE}

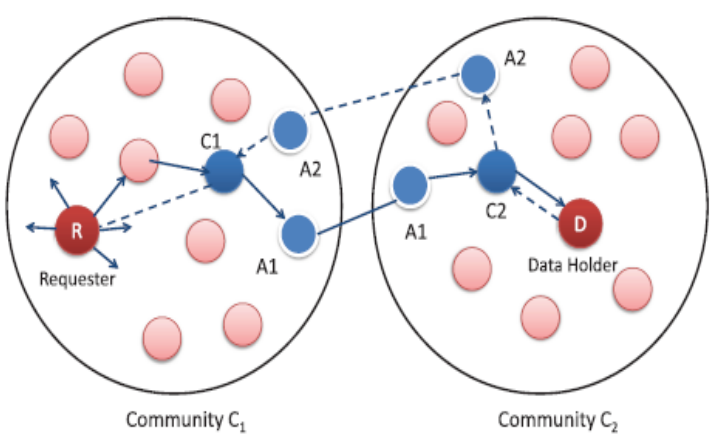

Figure 2.1 System architecture

\subsection{Interest mining}

Without loss of all inclusive statement, we expect that hub substance can be grouped to diverse interest classifications. It was discovered that clients more often than not have a couple record classifications that they question for records regularly in a record imparting framework. In particular, for the dominant part of clients, 80 percent of their imparted records fall into just 20 percent of aggregate record classifications. Like other record imparting frameworks we consider that a hub's put away records can mirror its record intrigues. Hence, SPOON determines the hobbies of a hub from its records. Table 2 records the documentations utilized as a part of this area. To determine its intrigues, a hub induces catchphrases from each of its records utilizing the archive grouping method. In particular, a hub determines a record vector for each of its documents from its metadata. We receive the system in the content recovery writing to ascertain the heaviness of an essential word. In the wake of recovering the record vector of each of its records, a hub characterizes its records to infer its advantage Bunche. Since the similitudes among documents are known, we utilize the AGNES technique to group the records into vested parties in a progressive way. Every document shapes an individual gathering at first. At that point, two most comparable record gatherings are converged in every step. This methodology rehashes until the closeness between any two gatherings is beneath a limit. The comparability between two gatherings is ascertained in light of their advantage vectors presented underneath. Therefore, a document is ordered to one and only intrigue bunch furthermore, there is no cover among gatherings. Every gathering has various documents.

\subsection{Community Creation}

Interpersonal organization hypothesis uncovers that individuals with the same interest have a tendency to meet every now and again. By misusing this property, SPOON characterizes hubs with regular hobbies furthermore, continuous contacts into a group to encourage interest-based document seeking, as presented recent in Area 3.5. Hubs with numerous intrigues fit in with various groups. The group development can effortlessly be led in a brought together way by gathering hub intrigues and contact frequencies from all hubs to a focal hub. Be that as it may, considering that the proposed framework is for circulated detached MANETs, in which convenient data gathering and circulation is nontrivial, we further propose a decentralized strategy to guarantee the adaptively of SPOON in genuine environment.3.4 Node Role
Assignment A past study has demonstrated that in an informal organization comprising of portable clients, just an a piece of hubs have high degrees. We can frequently locate an imperative or prominent individual who coordinates individuals in a group in our everyday life. For instance, the school dignitary coordinates distinctive divisions in the school, and the division head unites with employees in the division. Therefore, we exploit distinctive sorts of hub portability for document offering. We characterize group organizer and envoy hubs in the perspective of an interpersonal organization. A group organizer is an essential and well known hub in the group. It keeps records of all documents in its group. Every group has one envoy for every known remote group, which serves as the extension to the group. The organizer in a group keeps up the $\mathrm{VC}$ of remote groups and comparing representatives to guide inquiries to envoys for intercommunity searching.

\subsection{Node task Assignment}

We can frequently get an essential or else accepted one who manages associates within a society in our day by day life. For instance, the institution principal organizes various branches in the institution, and also the branch head joins to lecturers in the branch. Hence, we get improvement of various kinds of node mobility for file distribution. We describe society manager and representative nodes in the sight of a social network. A society manager is an essential and well-liked node in the society. It remains directories of each and every one file in its society. Every society has individual representative for every well-known foreign society, which serves like a connection to the society. The manager in a society keeps the $\mathrm{VC}$ of foreign communities and subsequent representatives to map queries to representatives for intercommunity.

\subsection{Interests-Leaning File Searching and Recovery}

In interpersonal organizations, individuals normally have a couple document intrigues and their document visit design for the most part takes after a certain conveyance. Additionally, individuals with the same interest have a tendency to contact one another much of the time. In this way, hobbies can be a great direction for document seeking. Considering the connection among hub development design, people's regular hobbies, and their contact frequencies, we can course document appeals to record holders in view of hubs' frequencies of meeting diverse hobbies. At that point, the interest-situated record seeking plan has two steps: intracommunity and intercommunity looking. A hub first pursuits documents in its home group. On the off chance that the facilitator finds that the home group can't fulfill a demand, it dispatches the intercommunity looking and advances the appeal to a representative that will go to the outside group that matches the demand's advantage. An appeal is erased when now is the ideal time to-live (TTL) terminates. Amid the hunt, a hub makes an impression on another hub utilizing the interest-situated steering calculation, in which a message is constantly sent to the hub that is liable to hold or to meet the questioned decisive words. The recovered document is steered along the inquiry way or through IRA if the course lapses. 


\section{RESULTS AND DISCUSSTIONS}

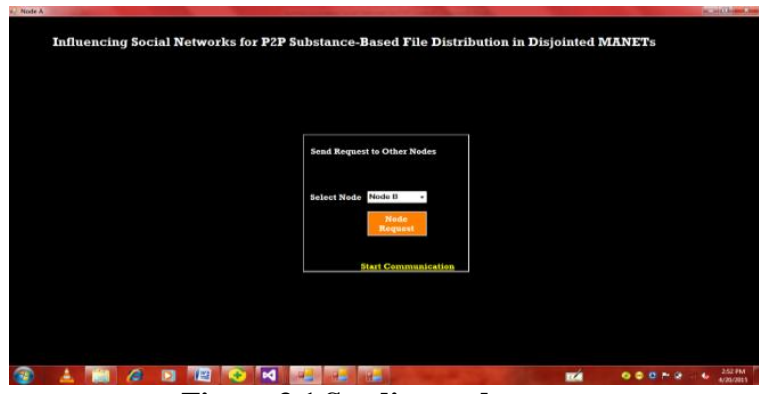

Figure 3.1 Sending node request

To start the communication, we need to select the node to which we send the file or receive the file.

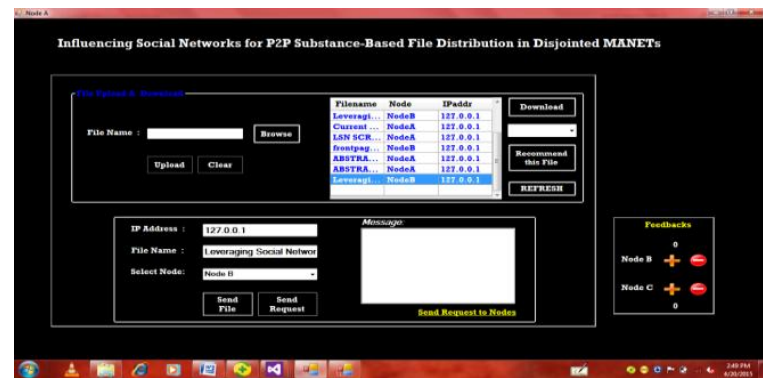

Figure 3.2 File Uploading, Sending File and Request

In this section, first we upload the file then by selecting the filename, ipaddress send request to other node. After receiving the request, send file to other node. Then we can download the requested file in the other node.

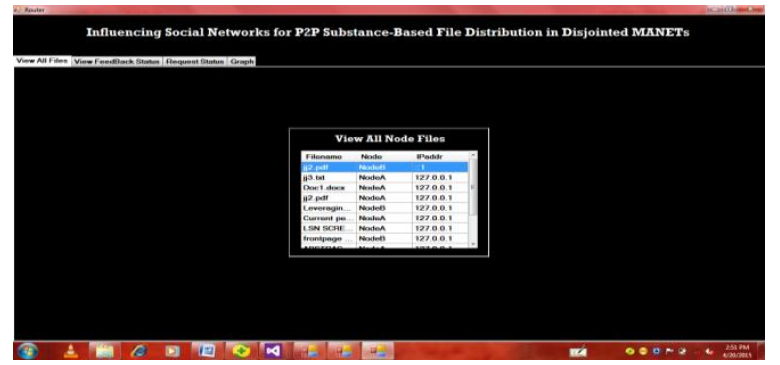

Figure 3.3 Viewing All Files, Feedbacks and Report Status

After executing all nodes, we can view all files, feedback status, Request status and graph which is based on our sending requests and files.

\section{CONCLUSIONS}

We introduced a social network-based Peer-Peer substance file distribution system in disjointed mobile ad hoc Networks. SPOON technology takes both node interest and contact occurrence for efficient file distribution. We proposed four major mechanisms of SPOON: Interest mining, which mean that identification of nodes' interests; area structure, constructs a familiar-interest nodes with common contacts into communities. The node task project element exploits nodes with fixed link with community members for intracommunity file probing and highly mobile nodes that call outer communities repeatedly for intercommunity file probing; The interest- leaning file probing method chooses forwarding nodes for queries supported on interest similarity. SPOON also include other strategies for file pre-fetching, querying-completion, and loop-prevention, and node churn consideration to develop further file probing effectiveness. The system operation on the genuine world GENI [Global Energy Network International] Orbit proposal and the tracedriven research confirm the effectiveness of SPOON. In future, we will discover how to establish suitable threshold in SPOON, and how they involve in the file distribution efficiency, and also how to adapt SPOON to better and more disjointed networks.

\section{ACKNOWLEDGEMENTS}

We take this opportunity to thank our project guide Mr.N.Sendhil Kumar and Mr.S.Palani for his valuable guidance and for providing all the necessary facilities, which were indispensable in completion of this paper.

\section{REFERENCES}

[1] A. Klemm, C. Lindemann, and O. Waldhorst, "A Special-Purpose Peer-to-Peer File Sharing System for Mobile Ad Hoc Networks," Proc. IEEE 58th Vehicular Technology Conf. (VTC '03), 2003.

[2] C. Lindemann and O.P. Waldhort, "A Distributed Search Service For Peer-to-Peer File Sharing,” Proc. Int'l Conf. Peer-to-Peer Computing (P2P '02), 2002.

[3] D.W.A. Hayes, "Peer-to-Peer Information Sharing in a Mobile Ad Hoc Environment," Proc. IEEE Sixth Workshop Mobile Computing Systems and Applications (WMCSA '04), 2004.

[4] J.B. Tchakarov and N.H. Vaidya, "Efficient Content Location in Wireless Ad Hoc Networks," Proc. IEEE Int'l Conf. Mobile Data Management (MDM ’04), 2004. 